\title{
La restauration et la réédition numérique de la musique
} Jean-Christophe Sevin

\section{Citer ce document / Cite this document :}

Sevin Jean-Christophe. La restauration et la réédition numérique de la musique. In: Culture \& Musées, n¹9, 2012. pp. 93-110;

doi : 10.3406/pumus.2012.1649

http://www.persee.fr/doc/pumus_1766-2923_2012_num_19_1_1649

Document généré le 26/01/2017 


\begin{abstract}
Restauration and digital republication of music.

Analysis of how physical and perceptual restoration and remastering of music recordings is based on an inquiry among restorers whose career and profile are used to explore the detail of the activity. The question that runs through this paper concerns the relationship between current listening norms and old processes of music recording, as well as the assemblage of old and modern technologies that are reissues represent. Practitioners who depend on a claim of equivalence between sound recordings and visual artworks to resist demands for changes that they consider too great in respect for their integrity can appear ambivalent in the assessment and treatment of these recordings. Their ambivalence finally reflects ordinary relationships with these recordings and the competing and conflicting values related to their appropriation.
\end{abstract}

\title{
Résumé
}

L'analyse des modalités matérielles et perceptives de la restauration et de la remasterisation des enregistrements musicaux est fondée sur une enquête auprès de restaurateurs dont la carrière et le profil sont mis à profit pour entrer dans le détail des activités. L'interrogation qui traverse ce travail concerne les rapports entre les normes d'écoute actuelles et les procédés d'enregistrements musicaux anciens, ainsi que l'assemblage de technologies anciennes et actuelles que constituent les rééditions. Les opérateurs qui s'appuient sur une mise en équivalence des enregistrements sonores avec les oeuvres plastiques, pour résister à des demandes de modifications qu'ils jugent trop importantes en regard du respect de leur intégrité, peuvent paraître ambivalents dans les évaluations et les traitements de ces enregistrements. Leur ambivalence renvoie finalement à celle des rapports ordinaires à ces enregistrements, aux valeurs concurrentes et contradictoires liées à leur appropriation.

\section{Resumen}

Restauraciones y reediciones digitales de los registros musicales.

El análisis de las modalidades materiales y perceptivas de la restauración y de la remasterización de los registros musicales está basado sobre una investigación entre los restauradores cuyas carreras y perfiles son ensalzados para profundizar en el detalle de sus actividades. La interrogación que atraviesa el trabajo concierne los vínculos entre las normas de escucha actuales y los procedimientos antiguos de grabación, así como el ensamblaje de tecnologías antiguas y actuales que constituyen las reediciones. Los operadores que se apoyan en la equivalencia de los registros sonoros con las obras plásticas, para resistir a las demandas de modificación que ellos estiman muy importantes en relación al respeto de su integridad, pudiendo así parecer ambivalente en las evaluaciones y tratamiento de esos registros. Su ambivalencia remite finalmente a los vínculos corrientes con esos registros, a los valores concurrentes y contradictorios ligados a su apropiación. 


\title{
LA RESTAURATION ET LA RÉÉDITION NUMÉRIQUE DE LA MUSIQUE
}

\begin{abstract}
L' a entraîné au cours de l'histoire la mise au point de techniques pour conserver, rendre disponible et (re)convocable la musique'. C'est par exemple lorsque l'on s'inquiète du transport et de la conservation des répertoires vocaux dans le rituel chrétien, à la période carolingienne (Hameline, 1982 : 25), que s'élaborent les premières solutions et procédures d'étalement graphique du son qui aboutissent à l'invention progressive de systèmes de notation codifiés, comme la portée et la partition.
\end{abstract}

On peut les considérer comme les premières formes de portabilité de la musique (Cheyronnaud, 2009). L'enregistrement sonore qui naît à la fin du XIX ${ }^{\mathrm{e}}$ siècle repose sur un autre principe en faisant appel à une chaîne de traduction qui prend en charge l'inscription sur un support, non plus de symboles représentant des sons, mais des sons eux-mêmes.

Cette phonographie était destinée au départ, dans l'esprit de ses concepteurs, à inscrire plus justement la parole que ne le fait l'écriture graphique (Tournes, 2008). Elle était ainsi vouée à des usages prioritairement administratifs et non à des usages musicaux. En tant que médium musical, le disque n'est pas né avec la mise au point de l'inscription physique des sons sur un support, il est le produit d'une invention collective, d'un travail de coproduction des acteurs qui s'en emparent : des producteurs et des industriels qui mettent la musique en répertoire et font du disque un produit de circulation rendant la musique accessible dans un nombre grandissant de foyers domestiques, des groupes d'amateurs qui 
définissent de nouvelles modalités et dispositions d'écoute du disque pour lui-même (Maisonneuve, 2009). C'est cet ensemble de transformations qui ont fait du disque un medium musical central dans le monde de la musique, tout comme le disque a luimême contribué à reconfigurer le monde musical.

La musique enregistrée peut être considérée comme un lieu de convergence entre art et technologie. Dès ses débuts l'enregistrement apparait comme un art du studio impliquant la maîtrise de sa gamme de sons et de timbres pour pouvoir " en jouer " (Sterne, 2005). Avec le développement des technologies d'enregistrement et des compétences nouvelles qui les accompagnent, la figure de l'ingénieur du son s'est imposée dans le contrôle de ce dispositif de création musicale (Horning, 2004). On peut se référer ici au rôle du producteur dans les musiques populaires, inventeur d'esthétiques nouvelles quand l'enregistrement s'écarte de la convention d'avoir à recréer l'illusion d'une reproduction fidèle d'une performance musicale réelle (Moorefield, 2005). Cette histoire de l'enregistrement, qui a maintenant plus d'un siècle, est riche en développements concernant les technologies d'enregistrement, les moyens de diffusion, les formats et les supports mais aussi les manières inattendues dont ils peuvent être appropriés par des acteurs collectifs ou individuels. Ce sont autant de changements qualitatifs qui à chaque fois reconfigurent notre relation à la musique. L'exemple le plus récent est l'impact des technologies numériques dans les domaines de la production et de la diffusion, mais aussi l'évolution massive des pratiques tournées vers la portabilité de la musique avec les formats de compression et l'augmentation des capacités de stockage et de diffusion en ligne.

La numérisation du son et l'apparition de nouveaux supports numériques entrainent également la nécessité - ou tout au moins l'opportunité - de conserver, de restaurer mais aussi de rééditer les enregistrements musicaux anciens. Nous changeons ici de perspective en abordant les rapports entre les dernières évolutions technologiques et des supports rendus obsolètes du fait même de ces évolutions qui compliquent l'accès aux enregistrements. Cet article examine les pratiques visant à faire migrer ces enregistrements dans une nouvelle configuration technique et culturelle. Il s'appuie sur une enquête réalisée auprès de cinq restaurateurs ${ }^{2}$ dont la carrière et le profil sont mis à profit dans la description détaillée et l'analyse des modalités pratiques de leurs activités, de leurs perceptions et de leurs jugements. Les rapports entre les normes d'écoute actuelles et les enregistrements musicaux anciens ainsi que l'assemblage de technologies anciennes et numériques pour les rééditions sont les interrogations qui traversent ce travail. 


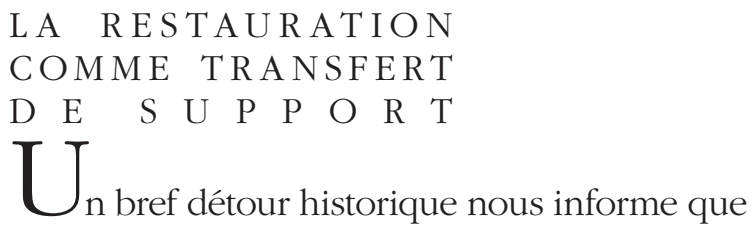

la constitution du patrimoine sonore passe d'abord par l'entreprise de conservation de la parole - avec par exemple la création des archives de la parole en 1911 - puisque c'est elle qui a d'abord été visée par l'enregistrement. Le premier signe de reconnaissance du caractère patrimonial de la musique enregistrée peut être repéré avec la création de la Phonothèque nationale en 1938, à laquelle revient la mission de collecte du dépôt légal des enregistrements sonores. L'accès à ces supports est cependant confronté à des obstacles juridiques, concernant aussi bien la reproduction que l'écoute, et techniques (Giuliani, 2004 : 124). Leur conservation implique en effet la nécessité d'anticiper la difficulté croissante de disposer des moyens de lecture de ces enregistrements. Le monde de l'enregistrement musical est ainsi marqué par une dynamique constante de renouvellement de ses technologies qui est en même temps une fabrique de l'obsolescence de ses dispositifs et de ses supports. De plus, ces derniers sont soumis à un processus de dégradation physico-chimique inéluctable qui, en dépit de bonnes conditions de conservation qui peuvent la différer, entraîne la nécessité de leurs transferts. Transférer un enregistrement analogique vers un support numérique implique un ensemble d'opérations comprenant l'extraction du son, sa conversion numérique et son inscription sur un nouveau support. Nous allons examiner de plus près l'opération d'extraction du son, en nous penchant sur le cas des enregistrements gravés sur disques analogiques - 78 tours ou 33 tours vinyle.

\section{LIRE UN DISQUE}

L'extraction de l'enregistrement est réalisée au moyen de la lecture du support. Les restaurateurs considèrent cette opération comme stratégique car elle conditionne la qualité des opérations suivantes. La lecture du support implique la maîtrise d'un ensemble de paramètres et de gestes liés à la chaîne d’intermédiaires dans laquelle circule le son. Le cas des disques 78 tours est éclairant car ils sont d'une époque où les technologies d'enregistrement n'étaient pas stabilisées. Cela oblige les restaurateurs à tenir compte de plusieurs types de paramètres tenant autant aux caractéristiques de la matière du disque qu'aux techniques de gravure, jusqu'aux caractéristiques du courant électrique - fréquence de secteur selon l'Europe ou les États-Unis - concernant la vitesse de leur gravure et, par conséquence, de leur lecture. 
Le disque 78 tours pouvant dater de plusieurs décennies, il faut tout d'abord vérifier son état de conservation et repérer la présence de corps étrangers qui peuvent encombrer les sillons. Le nettoyage de ce support a pour but de faciliter le passage de la pointe de lecture, pour lui permettre de restituer au mieux le signal sonore. Une technique courante consiste à humidifier le disque avec de l'eau déminéralisée afin d'atténuer le bruit de fond qui se manifeste lors de la lecture. Dans le cas de supports devenus extrêmement fragiles - disques à gravure directe par exemple -, une seule lecture peut être effectuée, sachant qu'elle condamne l'intégrité de l'original.

Le choix de la pointe de lecture s'avère très important car son adaptation à la forme et à l'état de surface du sillon de gravure conditionne largement la qualité du signal restitué. Or, la forme des sillons diffère selon les types de pressages, en fonction des différentes marques et selon les pays. Les pressages peuvent aussi changer pour une même marque, prenant en compte l'usure de la matrice en fonction du nombre de tirages et de la longue durée de vie du support 78 tours $^{3}$. Le restaurateur dispose donc d'une palette suffisamment étendue de pointes de lectures pour sélectionner celle qu'il juge avoir le meilleur rendu acoustique. Il effectue ainsi des tests d'écoute en fonction de critères liés à la forme et au degré d'usure du sillon : "Il faut choisir un bon diamant. On a une vingtaine de tailles, de types différents, coniques, elliptiques. Le tout c'est d'avoir un certain nombre de tailles parce que ça varie beaucoup entre les années vingt ou quarante ou cinquante, ce n'est pas du tout la même taille de gravure. Et encore ce n'est même pas forcément lié aux années. On peut avoir un enregistrement de février 1934 enregistré à Paris et un autre enregistré à Londres et avoir une bonne différence parce que le graveur n'était pas le même dans les deux villes. " (Entretien avec A. S.)

Un autre paramètre qui conditionne la qualité du rendu sonore du disque à partir de l'ère de l'enregistrement électrique est lui aussi exposé à une grande variabilité en fonction des marques et des époques : il concerne le réglage de la courbe de correction. Lors de la gravure d'un disque, le signal d'origine microphonique est converti sous la forme d'un sillon dont les grandeurs sont analogiques au signal électrique décrivant les évolutions du son. Mais, pour pallier les contraintes physiques rencontrées lors de la gravure des oscillations dans la cire en fonction de leurs fréquences - sons aigus, graves, etc. -, une amplification du niveau des fréquences aiguës et une atténuation de celui des fréquences graves sont appliquées. Une courbe de correction est appliquée en proportion inverse lors de la reproduction du son, à la lecture du support, afin de rétablir la balance spectrale. Or, avant que cette courbe ne soit standardisée avec les disques vinyles - 33 tours et 
45 tours - au milieu des années cinquante ${ }^{4}$, celle-ci variait de manière considérable selon les firmes et évoluait dans le temps au sein de chaque firme.

Les restaurateurs qui doivent déterminer cette courbe disposent parfois de repères comme les livrets recensant les diverses courbes utilisées selon les firmes et selon les années, ou bien de préréglages disponibles sur certains dispositifs de pré-amplification. Mais ils ne jugent pas ces repères suffisamment fiables pour servir d'unique ressource, ils mettent aussi en avant la nécessité de prendre en compte l'ensemble des paramètres impliqués : les caractéristiques de la gravure, l'état du sillon, les caractéristiques de la tête de lecture choisie, etc. Ils évoquent alors le "travail à l'oreille ", une écoute non guidée par ce type de repères, pour déterminer par essais et tâtonnements successifs la courbe qui restitue le mieux, selon eux, le signal sonore : "Les courbes ne sont pas toujours bonnes, alors on compense avec une machine, en rack qui est là [sur sa table de travail], dans laquelle on a préréglé les différentes courbes. Là il s'affiche sur RIAA, là on a la courbe BBC, la courbe Bin c'est l'allemande, d'une certaine période. " (Entretien avec J. F. P.) " On désactive la courbe et après on fait travailler l'oreille [...] c'est beaucoup à l'expérience. Avec les 78 tours, c'est le meilleur exemple, il n'y a aucune règle, chaque disque est complètement différent. Chaque enregistrement est différent. "(Entretien avec A. S.)

Le réglage de cette courbe repose ainsi sur la prise en compte de repères externes - livrets recensant les courbes, signes inscrits dans la matière du disque, usure, type de pointe de lecture - mais aussi sur un engagement sensoriel. Ce que les restaurateurs désignent comme "l'oreille " désigne un corps expert produit dans l'expérience, au fil des écoutes et des épreuves répétées, des essais et des erreurs, par lesquelles s'affine un rapport sensible à ces supports. Leurs compétences sont autant techniques qu'esthétiques. Il s'agit de la maîtrise technique des intermédiaires et des dispositifs permettant une forme de prévision dans le discernement du rendu sonore d'un enregistrement, dans la perception du matériau sonore.

Mais c'est aussi une relation esthétique qu'évoquent les restaurateurs. Celle-ci fait appel d'une part à une sensibilité et une attention aux singularités et d'autre part à une culture sonore et musicale. Prenons l'exemple de la tonalité qui est liée au réglage de la vitesse de lecture du disque. Dans le cas des disques 78 tours, elle n'est semble-t-il que rarement de 78 tours par minute, en raison de l'instabilité du courant électrique caractéristique de son époque de production. Ce réglage a des conséquences déterminantes, dans la mesure où la vitesse de lecture influe sur la tonalité, le diapason des voix. Un mauvais réglage peut ainsi changer la voix d'un 
chanteur, devenue par exemple plus aiguë ou plus grave. Pour les enregistrements parmi les plus anciens comportant des voix parlées, la variabilité de la vitesse de rotation - aucun repère n'est possible - est responsable d'une grande incertitude sur le rendu de la voix - hauteur, timbre, débit, etc.

\section{LA “ COPIE DROITE " \\ OU LA NEUTRALITÉ FORMELLE DU RESTAURATEUR}

Le transfert d'un enregistrement sonore met donc en jeu un ensemble de paramètres techniques, de gestes et de perceptions. Ce ne sont pas des intermédiaires neutres ou passifs mais autant de médiations (Hennion, 1993) dont chacune implique une action qui a des conséquences sur le son. Cela pourrait nous inciter à adopter une posture critique vis-à-vis de l'expression " copie droite " désignant le transfert de support dans les institutions de conservation - notamment l'INA et la BNF -, car elle peut évoquer l'idée de reproduction à l'identique. En effet, les opérations de transfert, nous l'avons vu, impliquent un ensemble de traductions et de transductions - conversions du son en signal électrique, données analogiques et numériques -, qui non seulement sont paramétrables mais changent en fonction du type de matériel utilisé. Lorsque l'on écoute les restaurateurs parler de ces opérations, la question du matériel est omniprésente. L'évocation récurrente et précise des machines - marque, modèle, année, etc. - ne relève pas d'une forme de fétichisme, mais provient du fait que chaque machine, chaque marque ou modèle a sa propre opacité, sa propre couleur et des résultats qu'ils apprennent à distinguer. Mais si chaque machine transmet différemment le son, chaque restaurateur a sa propre interprétation du son, sa façon de négocier entre les différents paramètres qu'il doit prendre en compte. Le restaurateur est lui-même un élément de cette chaîne dans laquelle le son circule, il " passe " à travers sa propre sensibilité et son écoute.

La " copie droite " n'est donc pas une notion adéquate pour décrire la réalité du transfert de support car celui-ci implique des modifications inévitables. Il faut néanmoins comprendre cette notion comme l'expression d'une convention, une manière de percevoir et de traiter le matériau sonore. La copie droite doit être comprise comme la meilleure lecture possible du support, celle qui est susceptible d'extraire le plus d'informations sonores sans introduire de biais de lecture. La neutralité de l'opération est formelle, au sens juridique du terme : elle ne concerne pas l'objet de l'activité, sa matière, mais la procédure mise en ouvre. L'extraction du son reste une interprétation du restaurateur, il joue de la platine mais de la façon la plus neutre qu'il puisse en juger. 


\section{LES ENREGISTREMENTS}

\section{$\begin{array}{lllllll}\text { A } & \mathrm{N} & \mathrm{C} & \mathrm{I} & \mathrm{E} & \mathrm{N} & \mathrm{S}\end{array}$ \\ $\mathrm{S} \mathrm{O} \mathrm{N} \mathrm{T}-\mathrm{I} \mathrm{L} \mathrm{S}$ \\ A MÉLIOR A B L E S? \\ L' \\ extraction du son par la lecture du support}

est une opération commune aux domaines de la conservation et de la réédition mais dans ce dernier cas elle ne représente qu'une étape. Celle-ci est primordiale car elle conditionne la qualité des interventions ultérieures qui ne pourront pallier une lecture jugée insatisfaisante, cependant elle reste une base de travail à partir de laquelle des interventions complexes seront effectuées.

Les rééditions étaient déjà pratiquées avec les supports analogiques, par exemple les 78 tours réédités en 33 tours vinyles. Mais c'est l'avènement du CD (compact disc) dans les années quatrevingt qui a entraîné un large mouvement de réédition des enregistrements analogiques sur ce support numérique. Ce mouvement doit aussi son importance à un changement de cadre législatif. En effet, depuis la loi de juillet 1985, dite " loi Lang ", les droits patrimoniaux de l'artiste interprète ou du producteur de phonogramme expirent cinquante ans après le $1^{\text {er }}$ janvier de l'année civile suivant la première publication, les rendant ainsi disponibles pour de nouvelles éditions ${ }^{5}$ dans la mesure où ils tombent dans le domaine public. Une réédition est engagée par un éditeur ou un producteur pour lesquels il s'agit de réintroduire un enregistrement musical dans le circuit commercial. Ils s'adressent généralement à un prestataire pour extraire l'enregistrement du support d'origine et le traiter en vue de réaliser un nouveau master, c'est-à-dire un nouvel original à partir duquel seront pressés des exemplaires. Dans cette perspective il s'agit moins de transfert de restauration que de remasterisation. Il est cependant utile de maintenir la catégorie analytique de restauration parce qu'elle implique les notions d'authenticité et de respect de l'intégrité de l'œuvre, ce qui nous permettra de questionner les interventions effectuées sur les enregistrements sonores.

Les restaurateurs/remasteriseurs que nous avons suivis ont vécu au cour de leur activité la transition entre les technologies analogiques et les technologies numériques, notamment le développement progressif des possibilités de traitement du signal sonore par des procédés algorithmiques installés dans des machines informatiques. Mais leur culture technique du monde de l'édition phonographique et de l'évolution des technologies d'enregistrement est indissociable d'une culture musicale qu'ils jugent nécessaire pour le traitement du matériau sonore de l'œuvre ou de l'artiste enregistré. Leur suivi permet de mettre au jour la tension entre leur statut de prestataire du monde de l'édition phonographique 
et leur attachement au respect de l'intégrité sonore des enregistrements. Plus précisément, cette tension se manifeste entre les éditeurs ou les producteurs, ayant des attentes concernant le nettoyage du son, et ces opérateurs qui tentent de sensibiliser sur les conséquences d'opérations simplement techniques en apparence.

\section{LE CHANGEMENT TECHNOLOGIQUE \\ ET SES CONSÉQUENCES POUR LA RÉÉDITION}

Le nettoyage du son se rapporte à l'élimination des "bruits " générés par la lecture du support. Ces "bruits " se manifestent inévitablement avec les supports analogiques et leur importance a tendance à augmenter avec le temps et les lectures répétées. Les lectures successives entraînent en effet la déformation plastique des sillons, due au poids du stylet. Elles peuvent faire apparaître des aspérités, arracher un peu de matière ou encore provoquer des rayures au moindre incident de manipulation. De plus, l'effet du temps sur l'évolution chimique de la matière organique du disque peut être accéléré lorsque les conditions de stockage s'avèrent non satisfaisantes.

Ces micro-transformations du support génèrent donc à la lecture des bruits dits "impulsionnels ", c'est-à-dire des impulsions d'énergie acoustique ayant une courte durée, de l'ordre de la milliseconde. Ils sont plus familièrement nommés " clics " et sont très nombreux : on peut en compter plusieurs dizaines par seconde sur un disque 78 tours. Les craquements ou "scratches " sont un autre type de bruit localisé provenant de la dégradation physique du support. Ils se distinguent par leur plus longue durée et leur forte amplitude par rapport au niveau du signal enregistré. Ils sont plus saillants dans le cas des disques 78 tours mais ils se retrouvent aussi avec les vinyles. La nature différente de ces supports, en raison de leur composition matérielle et de leur trajectoire d'usage, amène ainsi ces bruits impulsionnels à se manifester différemment. C'est du moins comme cela qu'ils sont perçus par les opérateurs.

Ce phénomène de bruits liés à la lecture des supports anciens prend une importance particulière et un caractère problématique dans l'environnement technologique. Cela se rapporte aux habitudes d'écoutes différentes générées par l'usage des supports analogiques et numériques, ainsi qu'aux possibilités offertes par le traitement numérique du signal sonore et ses conséquences éventuelles.

Dans l'environnement analogique, des correcteurs et des filtres étaient déjà employés pour la réduction de souffle. Dans le cas de la bande magnétique, on avait aussi recours au grattage de la bande ou à des micro-coupures aux ciseaux pour éliminer les "scratches". Mais les filtres et les correcteurs manquaient de 
précision de sorte que leur emploi s'avérait limité. Tandis que les micro-coupures et les grattages sur bandes magnétiques comportaient l'inconvénient de détériorer la bande et étaient susceptibles d'amputer l'enregistrement de façon irréversible. En outre, les coupures de bandes effectuées afin d'éliminer les "scratches " pouvaient générer des phénomènes temporels gênants, en raison du fait que le signal ne retombait pas en phase (Lubin \& Risler, 1996). Par rapport à ces limitations, les technologies numériques offrent de réels progrès et permettent d'éviter ce type d'inconvénients.

Numériser le son permet en effet d'appliquer des traitements informatiques dont l'efficacité n'a cessé de croître. Le signal analogique étant traduit et codé en chiffres binaires, un ordinateur peut ensuite effectuer des opérations complexes très rapidement et appliquer divers algorithmes. C'est ainsi que des procédés utilisant le traitement numérique du signal ont été mis au point et sans cesse améliorés, également en raison de l'augmentation de la puissance de calcul des micro-processeurs, qui allaient permettre notamment de travailler en temps réel sur les enregistrements. Ainsi, si l'élimination des "scratches " et des " clics" produisent toujours une altération du signal sonore, ces données perdues pendant un très court instant sont ensuite resynthétisées par un calcul mathématique d'interpolation. Il est ainsi possible de supprimer l'effet d'une petite lacune dans la couche d'oxyde d'un enregistrement magnétique par exemple. De plus, la numérisation de l'enregistrement permet d'intervenir sur le son sans avoir à intervenir sur le support d'inscription de l'enregistrement, car les données numériques sont transférées sur les disques durs de stations informatiques avant d'être traitées par des algorithmes. C'est un avantage non négligeable car les interventions sur le son sont virtuelles, de sorte qu'il est toujours possible de les annuler en séance et de recommencer jusqu'à ce que le résultat soit jugé satisfaisant. En revanche, une fois validées, ces modifications deviennent irréversibles, sauf si une copie de sauvegarde a préalablement été effectuée. Les opérateurs acquièrent ainsi une plus grande souplesse de travail et peuvent mener des micro-interventions et faire des micro-montages plus aisément, sans avoir à intervenir sur les supports eux-mêmes. Les technologies numériques ont l'autre avantage d'offrir des représentations graphiques détaillées du son, ce qui permet à l'opérateur de disposer d'un appui visuel utile pour identifier et sélectionner les fragments sur lesquels il intervient.

Chez les amateurs et usagers ordinaires de la musique enregistrée, l'avènement des supports numériques a contribué à installer une norme d'écoute différente car ils font disparaitre les bruits de contacts qui se manifestaient à l'écoute des supports analogiques - bandes magnétiques et disques 78 ou 33 tours. Ces phénomènes 
acoustiques apparaissaient en effet comme normaux du fait qu'ils étaient inhérents à l'écoute de la musique enregistrée. Alors qu'ils étaient relativement inaperçus, ils deviennent plus saillants dans un environnement numérique où les bruits de contact, non seulement ne sont plus habituels mais peuvent être perçus comme gênants, voire catégorisés comme un défaut des enregistrements analogiques. Cette norme d'écoute actuelle a des conséquences sur la réédition car le transfert des enregistrements analogiques sur supports numériques conserve les bruits - souffle, clics, etc. - liés à leur lecture. Or, selon les restaurateurs/remasteriseurs, les éditeurs et producteurs qui sont leurs commanditaires peuvent avoir tendance à privilégier un son propre aux dépens de sa richesse harmonique.

\section{LES ENREGISTREMENTS ANCIENS EN DÉFAUTS}

La suppression des bruits de contact a des conséquences sur la richesse harmonique du son, elle entraîne inéluctablement la suppression d'une partie de l'information sonore enregistrée et par conséquent d'une partie de son intégrité. Pour les restaurateurs, " enlever du souffle c'est aussi enlever du son " (Entretien avec L. R). Ils tentent ainsi d'infléchir les attentes de certains éditeurs pour correspondre aux nouveaux standards du son propre et sans aspérités que les supports numériques ont promus. Ils évoquent des compromis avec l'éditeur entre la préservation de l'intégrité de l'enregistrement et l'élimination des bruits les plus saillants à l'écoute. Cela demande un traitement en finesse du matériau sonore, des interventions localisées, "au stylo ", à l'aide de la représentation graphique du son. Mais ces opérations minutieuses demandent du temps et augmentent en conséquence le budget que l'éditeur devra allouer au prestataire. Le compromis est donc aussi d'ordre budgétaire. Celui-ci peut par exemple se manifester par des opérations standardisées avec un traitement automatique et paramétré du bruit de fond lié au contact de l'aiguille sur le support, et un traitement des " clics " et des "scratches " réalisés de façon localisée. Une remasterisation attentive au respect du son orignal est donc conditionnée, non seulement par la décision du commanditaire, convaincu ou non de la nécessité d'un compromis mais aussi, et peut-être surtout, au budget que celui-ci peut lui consacrer. En fonction des éditeurs, le budget d'une remasterisation peut être jugé de plus ou moins grande importance à l'aune de celui des autres opérations impliquées par une réédition - maquette, communication, distribution, etc. Le paysage de la réédition semble ainsi contrasté. Si les enregistrements bénéficient d'une forme de prestige ou de reconnaissance, ce budget pourra être conséquent, tandis que les enregistrements moins connus ou reconnus verront ce budget réduit. Dans ce cas, un usage 
principalement automatisé des procédés de traitements numériques sera effectué avec un minimum d'utilisation manuelle et localisée.

Pour les restaurateurs/remasteriseurs, les injonctions au " nettoyage du son " représentent un danger d'autant plus réel que les possibilités offertes par les outils numériques de traitement du signal sont susceptibles d'entraîner des modifications importantes : "On arrive dans les cas de traitement important, comme ceux réalisés avec le système No Noise, à un son qu'on peut qualifier de manière analogique à un "son carton", c'est-à-dire un son qui est nettoyé, mais dont les harmoniques ont disparu. On a l'impression que les trompettes ne sont plus en cuivre mais en carton, il n'y a plus de dynamique. De plus, toutes les micro-informations de réverbération naturelle s'effacent. "(Lubin \& Risler, 1996 : 65.) Ils tentent aussi de résister à la pression de certains éditeurs ayant tendance à vouloir améliorer ces enregistrements à l'aide des technologies numériques. Par exemple en faisant appel à des traitements qui recréent des harmoniques afin de donner l'illusion que l'enregistrement est récent, c'est-à-dire enregistré avec des moyens actuels. La volonté de valoriser l'enregistrement comporte le risque, pour les opérateurs, de lui faire perdre ce qui fait sa "vraie valeur ", en aboutissant à quelque chose "qui n'a rien à voir avec la voix originale de M. Trenet, ou le son du Steinway qu'utilise Rubinstein tel jour " (entretien avec J. F. P.).

Pour justifier leur désaccord, ils effectuent une montée en généralité (Boltanski \& Thévenot, 1991) pour rapprocher leur cas de celui du traitement des arts plastiques. La mise en équivalence avec la restauration en peinture leur permet de faire apparaitre les propositions de modification des enregistrements comme étant incongrues, on ne "repeint pas la Joconde " : "On peut mettre aussi de la peinture acrylique sur la chapelle Sixtine, c'est pas un problème. C'est ce que je leur ai dit : donnez-moi de la peinture acrylique et je vous refais la Joconde, je la trouve un peu terne." (Entretien avec J. F. P.)

Les critiques émises par les opérateurs concernent d'une part la trop grande confiance dans les technologies numériques, et d'autre part la trop grande valorisation des opérations techniques au détriment de l'approche esthétique. Ils reconnaissent que les procédés numériques ont permis une amélioration de leur travail mais ils restent critiques sur la toute-puissance des procédés numériques, parfois entretenue dans la littérature technique et commerciale, au motif qu'elle peut amener à pratiquer des opérations de traitement du signal sonore qui sortent du cadre de la restauration : "Un jour on me disait "on peut maintenant récréer les harmoniques artificiellement pour donner l'impression que l'enregistrement est récent". Non, parce qu'on ne connait pas le timbre exact, on va détimbrer, on va changer le timbre de l'instrument ou de la voix 
[...] Bien sûr il y a des commerçants qui vont vous dire qu'on arrive à faire ça, on fait des miracles avec les commerçants. "(Entretien avec J. F. P.)

Ces critiques sont aussi une façon, pour les restaurateurs, de dénoncer le manque de reconnaissance de leur activité, dont la perception se réduirait à la dimension technique. Dans les récits qu'ils font de leurs parcours, ils mettent ainsi en avant l'importance de l'acquisition d'une culture musicale et d'une "oreille " capable de discerner finement les caractéristiques de la matière sonore en fonction des machines et supports. Ce type de compétence leur apparaît comme étant d'autant plus nécessaire que les outils numériques utilisés pour les opérations de remasterisation sont capables d'entraîner des modifications importantes par rapport à l'enregistrement original. Cependant, si la reconnaissance des compétences des restaurateurs/remasteriseurs paraît incertaine, le statut des enregistrements musicaux ne semble pas stable non plus. Cela transparait dans leurs propos lorsqu'ils évoquent certaines opérations effectuées sur les enregistrements. En effet, si l'on maintient la mise en équivalence qu'ils effectuent entre le respect de l'authenticité d'un tableau et celui d'un enregistrement ancien, leur position peut paraitre ambiguë. Par exemple lorsque l'un d'entre eux évoque une intervention visant à pallier les conditions techniques précaires et les carences des technologies anciennes en intervenant sur l'égalisation, sur la mise à niveau des différentes pistes, afin de "retrouver un son naturel " (entretien avec L. R.). Ou lorsqu'un autre raconte comment il tente de remédier à une " mauvaise prise de son" (entretien avec J. F. P.) en intervenant sur le niveau de l'enregistrement pour le rehausser.

Lambivalence du statut des enregistrements anciens réédités en numérique peut ainsi être repérée dans la tension entre la neutralité formelle de l'opérateur qui tente de se conformer à l'intention esthétique qui préside une prise de son et ce qui peut apparaître comme une forme d'actualisation conforme à une esthétique sonore contemporaine. Il s'agit alors, en " redonnant un peu de jeunesse " (entretien avec L. R.) à l'enregistrement, de jouer sur les nombreux outils dits d'effets qui sont à disposition : reprise de la balance spectrale - paramétriques -, augmentation de la dynamique, ajout d'harmoniques, effet de salle - réverbération -, etc.

La catégorisation de certaines caractéristiques des enregistrements anciens comme des défauts s'avère pourtant relative. En effet, si les défaillances techniques qu'ils sont susceptibles de révéler à l'écoute peuvent être considérées comme des défauts, leurs carences ou limitations techniques sont surtout identifiées depuis les développements technologiques ultérieurs qui ont permis d'améliorer la capture et le traitement du son. Le matériel d'enregistrement pouvait avoir des défaillances, par exemple les irrégularités de rotation du 
plateau lors de l'enregistrement qui entraînent un " pleurage ", c'està-dire la déformation de la musique lors de sa lecture en raison d'une fluctuation initiale de la vitesse. Mais faut-il considérer comme des défauts le bruit ambiant du local dans lequel la musique a été enregistrée ou le bruit engendré par le burin graveur que l'on peut ensuite percevoir à l'écoute des disques ? On peut estimer que l'écoute de l'enregistrement dans son environnement technique d'époque, lu sur des équipements d'époque, avec une technologie de reproduction du son lui correspondant, ferait disparaittre ces défauts ${ }^{6}$. Non seulement ce type d'expérience est difficilement accessible mais on reste prisonnier d'une approche purement technique et l'on oublie que les dispositions esthétiques ordinaires envers la musique enregistrée ont elles-mêmes changé. Sauf à développer une posture savante ou d'amateur disposé à tirer plaisir des artefacts sonores - bruits, effets étrangers aux sons musicaux que produisent les enregistrements analogiques.

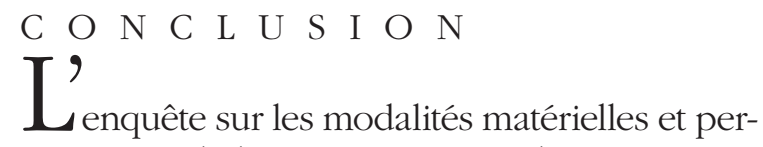
ceptives de la restauration et de la remasterisation des enregistrements musicaux nous amène à une interrogation sur les rapports entre l'ancien et l'actuel à travers l'analyse de cet assemblage de technologies numériques et d'artefacts musicaux rendus obsolètes par celles-ci. La reprise d'enregistrements analogiques avec des moyens techniquement incomparables et dans des formats et des standards actuels apparaît en effet comme un montage complexe. La musique enregistrée qui se trouve dissociée de son support d'origine tire de celui-ci une partie de ses caractéristiques mais elle doit subir une forme d'adaptation. Le transfert des enregistrements sur des supports numériques, loin de l'objectivité que pourrait laisser supposer l'idée d'une reproduction mécanique, fait apparaître un entrelacs de médiations techniques et humaines dès lors qu'on s'y intéresse de près. L'ensemble des éléments du dispositif dans lesquels le son circule entraîne inévitablement une modification de celui-ci en fonction des caractéristiques de chacun. Ce dispositif s'apparente ainsi à un instrument dont joue le restaurateur plus qu'il n'exécute des commandes mécaniques. Dans ce sens, la lecture d'un disque est une forme d'interprétation dont la neutralité est formelle, ainsi que nous l'avons vu.

La remasterisation désigne une opération plus ambitieuse que le transfert de restauration car elle consiste à produire une nouvelle matrice destinée à réinstaller l'enregistrement dans les circuits de la consommation musicale et dans cette perspective elle implique une forme d'adaptation à ses normes sonores. L'incertitude 
ontologique de l'enregistrement musical est mise au jour à travers l'analyse du traitement de ses faiblesses techniques, par contraste avec les possibilités actuelles, et des tentatives de corrections des défauts qu'on leur attribue. Les opérateurs qui s'appuient sur une mise en équivalence des enregistrements sonores avec les ouvres plastiques, pour résister à des demandes de modifications qu'ils jugent trop importantes au regard du respect de leur intégrité, peuvent aussi paraître ambivalents dans les évaluations et les traitements de ces enregistrements. Mais l'ambivalence des restaurateurs/remasteriseurs renvoie plus largement à celle des rapports ordinaires à ces enregistrements, aux valeurs concurrentes et contradictoires liées à leur appropriation. On peut ainsi privilégier l'enregistrement et sa valeur d'histoire (Riegl, 2003), en tant que document témoignant d'un état des interprétations musiciennes comme des techniques d'enregistrement. Une sonorité d'époque préservée n'est ici pas dissociable de l'appréciation de "la musique ". Mais on peut chercher la satisfaction dans l'écoute d'un artiste ou d'une ouvre, sans considération pour la valeur d'histoire de l'enregistrement et de la sonorité d'origine. Ainsi les "améliorations " sonores n'entravent pas mais facilitent l'accès à l'œuvre par un aménagement de son écoute. Si l'enregistrement sonore est un artefact historique c'est aussi son appréciation qui peut se déployer dans un mode historique. La prise de conscience de la distance technique et culturelle qui sépare le temps de la production de celui de la réédition, en tant que condition de l'appréciation, est concurrente avec l'ignorance de cette distance dans des versions actualisées ou mises au goût du jour ${ }^{8}$. Dans la configuration instable des rééditions d'enregistrements musicaux, ces deux versions cohabitent de façon plus ou moins pacifique.

\author{
J.-C. S. \\ Centre de la Vieille-Charité, \\ Marseille-Centre Norbert Elias (UMR 8562)
}

Manuscrit reçu le : 19 avril 2010.

Version révisée, reçue le : 28 novembre 2011.

$2^{\mathrm{e}}$ version acceptée, reçue le : 5 février 2012.

Article accepté pour publication, le : 16 février 2012. 
1. Ce travail n'aurait pas vu le jour sans Jean-Marc Fontaine (Institut d'Alembert, équipe LAM-université Pierre-etMarie-Curie). Ce travail a bénéficié de son expérience du monde des supports d'enregistrements et de leur restauration, de ses connaissances techniques aussi bien que de son ouverture aux problématiques des sciences humaines.

2. Une série d'entretiens et d'observations en studio a été effectuée entre 2007 et 2010 chez ces professionnels, dont quatre d'entre eux ont exercé d'autres métiers dans l'industrie du disque depuis les années soixante-dix.

3. De la fin du XIX ${ }^{e}$ siècle jusqu'à la moitié du XXe siècle.

4. Sous l'appellation de courbe RIAA, du nom de l'association interprofessionnelle de l'industrie du disque qui l'imposa : Recording Industry Association of America.

5. Quant au droit d'auteur il s'éteint à la fin de la soixante-dixième année civile qui suit l'année du décès de l'auteur de l'œuvre musicale.

6. C'est l'hypothèse que fait Nicola Mazzanti (2004) dans le cadre de la restauration du son au cinéma.

7. Cf. J.-L. Fabiani (2010) pour le cas du jazz et l'ambivalence des relations entre ses lieux de diffusions, ses dispositifs d'appréciation et ses enregistrements.

8. Cf. Morange, Dubois et Fontaine (2007) sur les appréciations différenciées d'une série de rééditions d'enregistrements de Caruso. Sur la prise en compte de la dimension historique dans la construction d'une relation actuelle aux " musiques anciennes ", voir Hennion (2011).
Boltanski (Luc) \& Thévenot (Laurent). 1991. De la justification: Leséconomies de la grandeur. Paris : Gallimard.

Brandi (Cesare). 2001. Théorie de la restauration. Paris : Monum-Éd. du Patrimoine.

Cheyronnaud (Jacques). 2009. " Rebuts de sons. "Bruit" comme terme de critique perceptive ". Ethnographiques. org. Publication en ligne : http:// www.ethnographiques.org/2009/ Cheyronnaud. Consulté le 5 mars 2012.

Fabiani (Jean-Louis). 2010. " Live at the Village Vanguard. Le paradoxe de l'écoute enregistrée du jazz ". L'Année sociologique, 60 (2), p. 387-402.

Fontaine (Jean-Marc). 2001. " De la restauration des documents sonores. Les disques noirs : Accès, dégradation, restauration ". Coré, 10, p. 36-46.

Giuliani (Elizabeth). 2004. "Les sources sonores du patrimoine musical " in Actes du colloque : À portée de notes: Musiques et mémoire, Grenoble, du 14 au 15 octobre 2003. Grenoble: Éd. ARALD-FFCB-Bibliothèques municipales de Grenoble.

Hameline (Jean-Yves). 1982. "Le chant grégorien ", p. 19-46, in Histoire de la musique. La musique occidentale du Moyen Âge à nos jours / sous la direction de Marie-Claire Beltrando-Patier. Paris : Bordas.

Hennion (Antoine). 1993. La Passion musicale. Une sociologie de la médiation. Paris : Métailié.

Hennion (Antoine). 2011. «Présences du passé : Le renouveau des musiques "anciennes" ". Temporalités. Publication en ligne : http://temporalites.revues. org/1836. Consulté le 12 mars 2012.

Horning (Susan Schmidt). 2004. " Engineering the Performance : Recording Engineers, Tacit Knowledge, and the Art of Controlling Sound ". Social Studies of Science, 34(5), p. 703-731.

Latour (Bruno). 2006. Changer de société. Refaire de la sociologie. Paris : Éd. La Découverte.

Lubin (Jacques) \& Risler (Lionel). 1996. "La restauration du son". Sonorités. Cahiers du patrimoine sonore et 
audiovisuel, 2, p. 63-69. [Actes des journées d'étude organisées par l'AFAS sous l'égide de la BnF les 26 et 27 mai 1994.]

Maisonneuve (Sophie). 2009. L'Invention $d u$ disque, 1877-1949. Genèse de l'usage des médias musicaux contemporains. Paris : Éd. des Archives contemporaines.

Mazzanti (Nicola). 2004. "Restoring Sound: Which Sound to Restore?", p. 224-233, in Le Son en perspective: nouvelles recherches / sous la direction de Dominique Nasta \& Didier Huvelle. Bruxelles : Peter Lang-Presses interuniversitaires européennes.

Moorefield (Virgil). 2005. The Producer as Composer: Shaping the Sounds of Popular Music. Cambridge : MIT Press.

Morange (Séverine), Dubois (Danièle) \& Fontaine (Jean-Marc). 2007. "Voices of Caruso : Cognitive Evaluation and Acoustic Analysis of Reedited Editions". Proceedings of the third Conference on Interdisciplinary Musicology. Publication en ligne : http:// www.uni-graz.at/ parncutt/cim07/ CIM07\%20Proceedings/CIM07_Morange-Dubois-Fontaine_Voices $\% 20$ of\%20Caruso.pdf. Consulté le 12 mars 2012.

Riegl (Aloïs). 2003. Le Culte moderne des monuments. Paris : Éd. L'Harmattan.

Sterne (Jonathan). 2003. The Audible Past: Cultural Origins of Sound Reproduction. Durham : Duke University Press.

Sterne (Jonathan). 2005. "Pour en finir avec la fidélité. Les médias sont des instruments ". Mouvements, 42, p. 44-53. Tournes (Ludovic). 2008. Du phonographe au MP3 : Une histoire de la musique enregistrée. Paris : Éd. Autrement. 


\section{¿analyse des modalités matérielles et percep-}

tives de la restauration et de la remasterisation des enregistrements musicaux est fondée sur une enquête auprès de restaurateurs dont la carrière et le profil sont mis à profit pour entrer dans le détail des activités. L'interrogation qui traverse ce travail concerne les rapports entre les normes d'écoute actuelles et les procédés d'enregistrements musicaux anciens, ainsi que l'assemblage de technologies anciennes et actuelles que constituent les rééditions. Les opérateurs qui s'appuient sur une mise en équivalence des enregistrements sonores avec les ouvres plastiques, pour résister à des demandes de modifications qu'ils jugent trop importantes en regard du respect de leur intégrité, peuvent paraître ambivalents dans les évaluations et les traitements de ces enregistrements. Leur ambivalence renvoie finalement à celle des rapports ordinaires à ces enregistrements, aux valeurs concurrentes et contradictoires liées à leur appropriation.

Titre: La restauration et la réédition numérique de la musique.

Mots-clés : Restauration, édition numérique, enregistrements musicaux, écoute, authenticité.

\section{1 nalysis of how physical and perceptual res-} toration and remastering of music recordings is based on an inquiry among restorers whose career and profile are used to explore the detail of the activity. The question that runs through this paper concerns the relationship between current listening norms and old processes of music recording, as well as the assemblage of old and modern technologies that are reissues represent. Practitioners who depend on a claim of equivalence between sound recordings and visual artworks to resist demands for changes that they consider too great in respect for their integrity can appear ambivalent in the assessment and treatment of these recordings. Their ambivalence finally reflects ordinary relationships with these recordings and the competing and conflicting values related to their appropriation.

Title : Restauration and digital republication of music.

Key words : Restoration, digital edition, music recordings, listening, authenticity.

\section{$\mathrm{E}$} análisis de las modalidades materiales y perceptivas de la restauración y de la remasterización de los registros musicales está basado sobre una investigación entre 
los restauradores cuyas carreras y perfiles son ensalzados para profundizar en el detalle de sus actividades. La interrogación que atraviesa el trabajo concierne los vínculos entre las normas de escucha actuales y los procedimientos antiguos de grabación, así como el ensamblaje de tecnologías antiguas y actuales que constituyen las reediciones. Los operadores que se apoyan en la equivalencia de los registros sonoros con las obras plásticas, para resistir a las demandas de modificación que ellos estiman muy importantes en relación al respeto de su integridad, pudiendo así parecer ambivalente en las evaluaciones y tratamiento de esos registros. Su ambivalencia remite finalmente a los vínculos corrientes con esos registros, a los valores concurrentes y contradictorios ligados a su apropiación.

Título : Restauraciones y reediciones digitales de los registros musicales.

Claves: Restauración, registros musicales digitales, escucha, autenticidad. 